\title{
Review of: "Characterization of chondroitin sulfate in stem cells derived from umbilical cord blood in rats"
}

\author{
Nicola Volpi ${ }^{1}$ \\ 1 Università degli Studi di Modena e Reggio Emilia
}

Potential competing interests: The author(s) declared that no potential competing interests exist.

Nicola Volpi

Department of Life Sciences, University of Modena and Reggio Emilia, Modena, Italy. e-mail: volpi@unimo.it

This study explores the presence, structure and some chemical properties of complex sulfated heteropolysaccharides, known as glycosaminoglycans (GAGs), chondroitin sulfate (CS) and heparan sulfate (HS) of hematopoietic stem cells (HSCs). In particular, the authors characterize CS and its isomeric form dermatan sulfate (DS) and HS present in mononuclear cells of rat umbilical cord blood cells (UCB-MNCs) as well as in their expanded stem-cell-enriched-UCBCs (SCE-UCBCs).

The principal finding was that the main glycosaminoglycan present in the rat UCB is a CS/DS hybrid polysaccharide mainly sulfated in position 4 of the $\mathrm{N}$-acetyl-galactosamine unit with a relatively low degree of sulfation. Moreover, a novel information derived from this study is the presence in the CS backbone, even if in very minor percentage, of the disaccharide named $\mathrm{E}$ disulfated in position 4 and 6 of the $\mathrm{N}$ acetyl-galactosamine residue. This polymer was detected in vascular intima and media of rat umbilical cord at embryonic day 19. HS was present but in a much less content than CS. The presence of this CS/DS hybrid biopolymer in UCB and derived stem cells was found related to its capacity to influence the stem cell proliferation and/or differentiation as in a colony-forming cell assay the percentage of cells decreased in culture when a CS degradation enzyme was applied.

Indeed, according to the international scientific literature, this study shows a number of methodological and analytical concerns below reported.

1. The presence of a low-sulfated CS in human placenta (https://doi.org/10.7554/eLife.65776) and in umbilical cord mesenchymal stem cells (https://doi.org/10.1074/jbc.M114.557447) is well known as well as its numerous biological capacities. In fact, these scientific knowledges are used to develop possible protection of malaria-infected pregnant women from placental malaria (https://doi.org/10.7554/eLife.65776) as well as in tissue development and in tissue repair and morphogenesis (https://doi.org/10.1002/stem.2860). Ultimately, the originality and novelty of this work are limited by the already multiple knowledges known in this topic.

2. No information is available to clarify the type(s) of proteoglycan(s) present in mononuclear cells of rat umbilical cord blood cells (UCB-MNCs) as well as in their expanded stem-cell-enriched-UCBCs (SCE- 
UCBCs) and made by CS and/or HS. This would have been particularly important in order to understand the possible activities of these macromolecules also considering that numerous other studies have characterized the types of proteoglycans present in stem cells and their activities (see for example https://doi.org/10.3390/ijms20194815).

3. No information is available on the presence and content of hyaluronic acid (HA) in mononuclear cells of rat umbilical cord blood cells (UCB-MNCs) as well as in their expanded stem-cell-enriched-UCBCs (SCEUCBCs). This is more important by considering that HA is involved in many cellular activities and functions. Moreover, the treatment used to reduce CS by means of the enzyme chondroitinase ABC is equally capable to degrade HA. Consequently, the decrease of the percentage of cells observed in culture in the reported colony-forming cell assay may be also due to the degradation of HA along with CS.

4. The disaccharide composition of the hybrid CS/DS has been evaluated by treatment with chondroitinase $A B C$ and $A C$ able to degrade this biopolymer in disaccharides and further their separation by HPLC. However, chondroitinase $A B C$ and $A C$ are also able to degrade HA in its constituent non-sulfated disaccharide that is not well separated by the reported HPLC approach and can co-elute with the nonsulfated disaccharide belonging to CS and DS. Consequently, the illustrated percentage of CS/DS nonsulfated disaccharide can also contain variable content of HA disaccharide.

5. The authors tested a CS type E from squid cartilage formed of about $60-65 \%$ of the disulfated disaccharide E in their CFU-GM assay. However, this makes little sense as the identified CS in umbilical cord blood cells and in their expanded stem-cell-enriched-UCBCs has a very low type E disaccharide content, of about $2-3 \%$.

6. Another concern of this work is the absence of fine characterization of the structure of the hybrid CS/DS polysaccharide, in particular the percentage of these two types of CS and of the two different types of uronic acids, glucuronic acid present in the CS and iduronic acid typical of the DS. This is important to understand if the biological activities are actually mediated by the CS or the DS. Similarly, the structure of HS has also not been well defined due to the lack of two other disaccharides in particular, the monosulfated disaccharide in C2 of the uronic acid residue and the disulfated disaccharide in position 2 of uronic acid and 6 of $\mathrm{N}$-acetyl- or N-sulfo-glucosamine.

By considering the above mentioned methodological and analytical concerns of the reported results, this publication largely appears as preliminary and more studies are needed to clarify the potential structural, physiological and potentially pathological role of these complex biomacromolecules on hematopoiesis in order to advance in this important topic of the glycobiology. 